\title{
Intraoperative cochlear nerve mapping with the mobile cochlear nerve compound action potential tracer in vestibular schwannoma surgery
}

\author{
Nobuyuki Watanabe, MD, ${ }^{1}$ Takuya Ishii, MD, ${ }^{2}$ Kazuhiko Fujitsu, MD, ${ }^{1}$ Shogo Kaku, MD, ${ }^{2}$ \\ Teruo Ichikawa, MD, ${ }^{1}$ Kosuke Miyahara, MD, ${ }^{1}$ Tomu Okada, MD, ${ }^{1}$ Shin Tanino, MD, ${ }^{1}$ \\ Yasuhiro Uriu, MD, ${ }^{1}$ and Yuichi Murayama, MD² \\ ${ }^{1}$ Department of Neurosurgery, National Hospital Organization, Yokohama Medical Center, Yokohama, Kanagawa; and \\ 2Department of Neurosurgery, Jikei University School of Medicine, Nishishinbashi, Tokyo, Japan
}

\begin{abstract}
OBJECTIVE The authors describe the usefulness and limitations of the cochlear nerve compound action potential (CNAP) mobile tracer (MCT) that they developed to aid in cochlear nerve mapping during vestibular schwannoma surgery (VSS) for hearing preservation.

METHODS This MCT device requires no more than 2 seconds for stable placement on the nerve to obtain the CNAP and thus is able to trace the cochlear nerve instantaneously. Simultaneous bipolar and monopolar recording is possible. The authors present the outcomes of 18 consecutive patients who underwent preoperative useful hearing (defined as class I or II of the Gardner-Robertson classification system) and underwent hearing-preservation VSS with the use of the MCT. Mapping was considered successful when it was possible to detect and trace the cochlear nerve.
\end{abstract}

RESULTS Mapping of the cochlear nerve was successful in 13 of 18 patients (72.2\%), and useful hearing was preserved in 11 patients (61.1\%). Among 8 patients with large tumors (Koos grade 3 or 4 ), the rate of successful mapping was $62.5 \%$ (5 patients). The rate of hearing preservation in patients with large tumors was $50 \%$ (4 patients).

CONCLUSIONS In addition to microsurgical presumption of the arrangement of each nerve, frequent probing on and around an unidentified nerve and comparison of each waveform are advisable with the use of both more sensitive monopolar and more location-specific bipolar MCT. MCT proved to be useful in cochlear nerve mapping and may consequently be helpful in hearing preservation. The authors discuss some limitations and problems with this device.

https://thejns.org/doi/abs/10.3171/2017.12.JNS171545

KEYWORDS vestibular schwannoma; cochlear nerve compound action potential; mapping; bipolar forceps; hearing preservation; oncology; surgical technique

$\mathrm{H}$ EARING preservation has become an increasingly important goal of vestibular schwannoma surgery (VSS) in patients who have useful hearing preoperatively. Several intraoperative neurophysiological methods have been developed for this purpose..$^{2-5,10,12,17,18} \mathrm{We}$ have developed a bipolar forceps-type electrode, called the cochlear nerve compound action potential (CNAP) mobile tracer (MCT), for cochlear nerve mapping. Simultaneous monopolar and bipolar recording is possible with this device. The MCT requires only a short time (no more than 2 seconds) for stable placement for summation of ac- quisitions and can thus trace the cochlear nerve instantaneously. In this paper we describe the results of 18 patients who underwent hearing-preserving VSS with the MCT, and discuss a way to map the cochlear nerve in combination with its presumptive anatomical location. The usefulness and limitations of the MCT are also discussed.

\section{Methods}

\section{Study Patients and Design}

Between January 2011 and December 2016, 61 consec-

ABBREVIATIONS ABR = auditory brainstem response; CNAP = cochlear nerve compound action potential; GR = Gardner-Robertson; HB = House-Brackmann; MCT = mobile CNAP tracer; PTA = pure tone average; SDS = speech discrimination score; VSS = vestibular schwannoma surgery.

SUBMITTED June 27, 2017. ACCEPTED December 4, 2017.

INCLUDE WHEN CITING Published online May 18, 2018; DOI: 10.3171/2017.12.JNS171545. 
utive patients underwent VSS through a retrosigmoid approach. Hearing preservation was attempted in 18 patients who had useful hearing preoperatively. Pre- and postoperative hearing was evaluated by the Gardner-Robertson (GR) classification system, ${ }^{6}$ which is based on the pure tone average (PTA) and the speech discrimination score (SDS). Useful hearing was defined as either class I or II (PTA $\leq 50 \mathrm{~dB}$ and SDS $\geq 50 \%$ ). Radiological tumor sizes were graded according to the Koos system. ${ }^{8}$ We divided the patients into two groups according to tumor size: the small-size group with Koos grade 1 or 2 tumors and the large-size group with Koos grade 3 or 4 tumors. Total removal of the tumor, including its intrameatal portion, was attempted in all 18 patients. In addition to continuous auditory brainstem response (ABR) monitoring, intermittent but constant tracing of the cochlear nerve was attempted with the MCT. We used Neuropack X1 MEB2312 (Nihon Koden) as the recording system from January 2011 to August 2015 and Neuromaster MEE-1216 (Nihon Koden) from September 2015 to December 2016. After inducing total intravenous anesthesia and placing the patient in the park bench position, a disc electrode (NE-113A, Nihon Koden) was placed on the earlobe of the affected side. The ground electrode was placed on the midline frontal zone.

\section{MCT Device}

The MCT has bipolar forceps-type electrodes made from bipolar coagulation forceps by remodeling their connecting terminals into terminals adapted to the recording system (Fig. 1). When the surgeon holds the forceps-type MCT and lightly brings its blades close together, the tips are fixed $1 \mathrm{~mm}$ apart by the rubber dampers. The tips of the MCT are placed on the nerve in parallel to the course of the nerve so that the surgeon can obtain a waveform as large and uniform as possible. ${ }^{1}$ The MCT can be used for simultaneous monopolar and bipolar recording. Monopolar recording is obtained from 1 of the 2 electrodes (anode), with reference to the ipsilateral earlobe electrode (cathode). Bipolar recording is obtained as the differential potential between the two electrode tips. The auditory stimulus was a 105-dB click sound delivered at a rate of 17 per second from the bilateral earphones. The potentials were filtered from 100 to $1500 \mathrm{~Hz}$. Each recording consisted of the summation of 30 acquisitions, which means that no more than 2 seconds was needed for stable placement of the MCT for each recording. In a typical waveform of the CNAP, the latency of the main negative peak occurred between 3.0 and $3.8 \mathrm{msec}$ after the onset of sound at a stimulus intensity of $105 \mathrm{~dB} .{ }^{9}$ With consideration of anatomical location and through analysis of the waveform, most of the time we were able to determine whether the given nerve was the cochlear nerve. We considered the mapping successful when it was possible to trace the entire course of the cochlear nerve with the MCT. In all cases, the facial nerve was monitored with the use of Nerve Integrity Monitor 3.0 response (Medtronic). Most VSSs were performed by K.F., and most MCT recordings were performed or supervised by $\mathrm{T}$. Ishii. Postoperative hearing examinations were performed approximately 2 weeks after surgery. The extent of tumor removal was also evaluated by postoper-

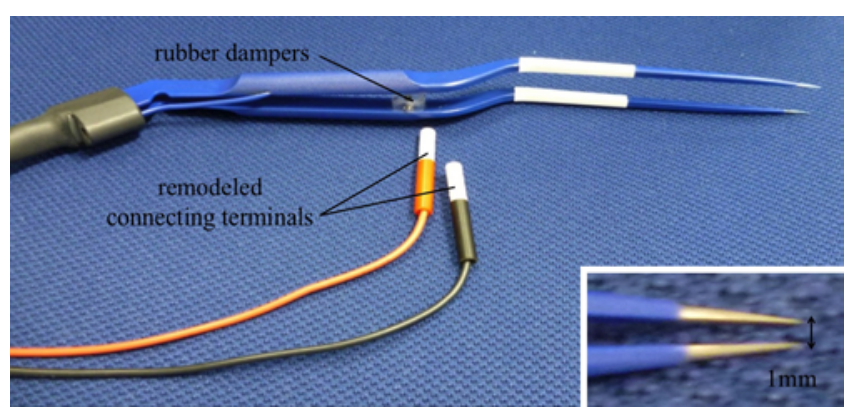

FIG. 1. A newly designed bipolar forceps-type electrode (MCT). The distance between the two electrode tips can be fixed at $1 \mathrm{~mm}$ (inset). Figure is available in color online only.

ative Gd-enhanced MRI around 2 weeks after surgery. Postoperative facial nerve function was graded according to the House-Brackmann (HB) grading system ${ }^{7}$ about 4 weeks and 12 months after surgery.

\section{Results}

Table 1 summarizes the characteristics of the 18 patients. There were 6 men and 12 women, with a mean age of $51.5 \pm 14.0$ years. Gross-total removal, including the intrameatal portion of the tumor, was achieved in all 18 patients. Postoperative facial nerve function evaluated approximately 4 weeks after surgery was $\mathrm{HB}$ grade 1 or 2 in 15 patients $(83.3 \%)$. At around 12 months, we were not able to evaluate 3 cases who had HB grade 1 or 2 at about 4 weeks. Three cases who had HB grade 3 or 4 at approximately 4 weeks postoperatively showed no significant improvement at around 12 months. Successful mapping of the cochlear nerve was achieved in 13 patients $(72.2 \%)$ at the final stage. ABR showed a similar tendency except in case 13, and is also presented in Table 1. Including this exceptional case, cases with failed MCT will be discussed in the discussion section below. Useful hearing was preserved in 11 patients $(61.1 \%)$. There were 10 patients in the small-size group (Koos grade 1 or 2 ) and 8 patients in the large-size group (Koos grade 3 or 4). In the small-size group, the rates of successful mapping and preservation of useful hearing were $80.0 \%$ (8 patients) and $70.0 \%$ (7 patients), respectively. In the large-size group, the rate of successful mapping was $62.5 \%$ (5 patients). The rate of hearing preservation in this group was $50 \%$ (4 patients).

\section{Illustrative Case (Large-Size Group)}

A 61-year-old woman (case 5) had a left vestibular schwannoma of Koos grade 4 (Fig. 2 left). Preoperatively, her hearing was GR class I (PTA $15 \mathrm{~dB}$, SDS 70\%). After substantial debulking of the ventrocaudal portion of the tumor, the MCT was placed on the root entry zone of the presumed cochlear nerve between the root exit zone of the facial nerve and the root entry zone of the presumed inferior vestibular nerve (Fig. 3). A triphasic waveform with a large peak at $3.5 \mathrm{msec}$ was recorded $(17.6 \mu \mathrm{V}$ by monopolar recording and $16.7 \mu \mathrm{V}$ by bipolar recording; Fig. 4A). From these findings, the nerve was recognized as the root entry zone of the cochlear nerve. After further debulking of the tumor, two nerves were found to be running parallel 
TABLE 1. Characteristics of the 18 patients who received hearing-preservation surgery with the MCT device

\begin{tabular}{|c|c|c|c|c|c|c|c|c|c|}
\hline \multirow[b]{2}{*}{ Case No. } & \multirow[b]{2}{*}{ Age (yrs) } & \multirow[b]{2}{*}{ Sex } & \multirow[b]{2}{*}{ Koos Grading } & \multicolumn{2}{|c|}{ GR Class } & \multirow[b]{2}{*}{ Mapping* } & \multirow[b]{2}{*}{$\mathrm{ABR} \dagger$} & \multicolumn{2}{|c|}{ Postop HB Grade } \\
\hline & & & & Preop & Postop & & & 4 Wks & $12 \mathrm{Mos}$ \\
\hline 1 & 59 & $\mathrm{~F}$ & 1 & I & I & Yes & Yes & 2 & 2 \\
\hline 2 & 69 & $M$ & 2 & 1 & I & Yes & Yes & 1 & 1 \\
\hline 3 & 48 & $M$ & 2 & ॥ & II & Yes & Yes & 1 & 1 \\
\hline 4 & 46 & $\mathrm{~F}$ & 3 & 1 & IV & Yes/no & Yes/no & 3 & 3 \\
\hline 5 & 61 & $\mathrm{~F}$ & 4 & 1 & I & Yes & Yes & 1 & 1 \\
\hline 6 & 51 & M & 3 & II & IV & Yes/no & Yes/no & 2 & ND \\
\hline 7 & 42 & $\mathrm{~F}$ & 1 & II & IV & Yes & Yes/no & 1 & 1 \\
\hline 8 & 72 & $\mathrm{~F}$ & 3 & 1 & I & Yes & Yes & 2 & 2 \\
\hline 9 & 59 & M & 2 & I & I & Yes & Yes & 1 & 1 \\
\hline 10 & 29 & $\mathrm{~F}$ & 2 & 1 & IV & No & Yes/no & 3 & 3 \\
\hline 11 & 24 & $\mathrm{~F}$ & 3 & 1 & 1 & Yes & Yes & 1 & ND \\
\hline 12 & 34 & $F$ & 3 & II & II & Yes & Yes & 1 & 1 \\
\hline 13 & 57 & $\mathrm{~F}$ & 1 & II & II & No & No & 1 & ND \\
\hline 14 & 76 & $M$ & 1 & 1 & I & Yes & Yes & 1 & 1 \\
\hline 15 & 43 & $M$ & 4 & 1 & IV & No & Yes/no & 4 & 3 \\
\hline 16 & 52 & $\mathrm{~F}$ & 3 & II & III & Yes & Yes & 1 & 1 \\
\hline 17 & 53 & $\mathrm{~F}$ & 2 & 1 & I & Yes & Yes & 1 & 1 \\
\hline 18 & 52 & $\mathrm{~F}$ & 2 & II & III & Yes & Yes & 2 & 1 \\
\hline
\end{tabular}

to each other, showing their dorsal aspects in the cerebellopontine angle cistern (Fig. 5). The deeper, rostral nerve was considered to be the cochlear nerve because of the larger amplitude ( $12.6 \mu \mathrm{V}$ at $3.5 \mathrm{msec})$ by bipolar recording (Fig. 4B right). The shallower one, in contrast, showed smaller waveforms $(3.8 \mu \mathrm{V}$ by monopolar and $3.9 \mu \mathrm{V}$ by bipolar recording; Fig. 4C). Therefore, the shallower nerve was considered to be the inferior vestibular nerve. This nerve was proved to be continuous with the vestibular fork (we call the approximating junction of the inferior and superior vestibular nerves near their root entry zones the "vestibular fork") at the later stage of tumor removal. Gross-total removal was achieved, including removal of the intrameatal portion of the tumor (Fig. 2 right) with successful mapping of the cochlear nerve. Postoperative hearing was GR class I (PTA $14 \mathrm{~dB}$, SDS 80\%).

\section{Illustrative Case (Small-Size Group)}

A 52-year-old woman (case 18) had a right vestibular schwannoma, and she presented with tinnitus lasting for a few months. MRI revealed an intrameatal tumor that showed a slight protrusion into the cerebellopontine angle cistern (Koos grade 2). The preoperative hearing level was GR class II (PTA $47.5 \mathrm{~dB}$, SDS 85\%). Caudal to and crossing over the facial nerve exit zone, two thin nerves ran close to each other over the ventrocaudal surface of the tumor. Both these nerves were so thin that the tumor capsule was visible through them. The deeper nerve had a large, clear peak at $3.6 \mathrm{msec}(10.3 \mu \mathrm{V}$ by monopolar and $7.5 \mu \mathrm{V}$ by bipolar recording) and was presumed to be the cochlear nerve (Fig. 6A). Combined with consideration of anatomical location, the other nerve was thought to be the inferior vestibular nerve because it exhibited no significant response (Fig. 6B). Bipolar recordings were attempted several times on several parts of the tumor surface substantially distant from these nerves. However, no significant response was obtained in any recording. In contrast, bipolar recording on the tumor capsule very close to but not exactly on the presumed cochlear nerve showed a slightly delayed response at $5.0 \mathrm{msec}$ with a considerably large amplitude (Fig. 6C). After a careful dissection of these nerves, gross-total removal of the tumor was performed. The final recording on the cisternal portion of the cochlear nerve exhibited a clear peak suggestive of CNAP (Fig. 6D). Cochlear nerve mapping was successful and ABR showed no remarkable change throughout the operation. However, the patient's postoperative hearing acu-
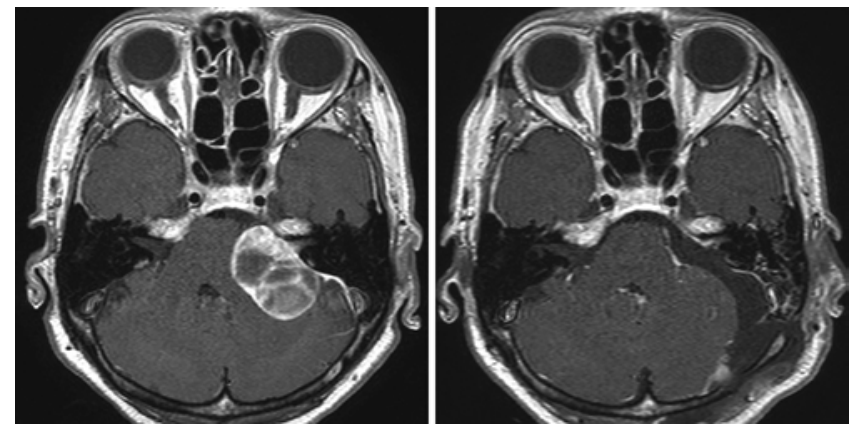

FIG. 2. Pre- (left) and postoperative (right) Gd-enhanced T1-weighted axial MR images in case 5. 


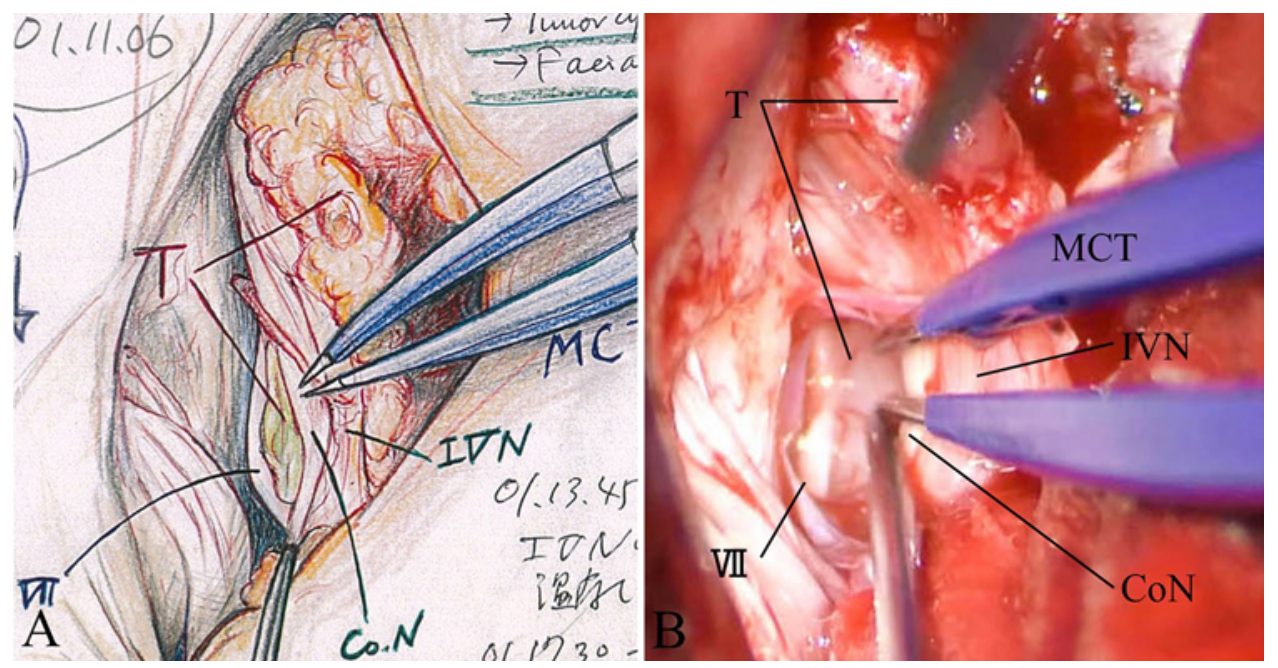

FIG. 3. Case 5. Operative note illustrated by the operator (A) and video capture (B). The MCT was placed on the ventral aspect of the cochlear nerve $(\mathrm{CoN})$ root entry zone. $\mathrm{MCT}$ recording is presented in Fig. $4 \mathrm{~A}$. IVN = inferior vestibular nerve; $\mathrm{T}=$ tumor; $\mathrm{VII}=$ facial nerve. Copyright Kazuhiko Fujitsu (panel A). Published with permission. Figure is available in color online only.

ity was downgraded to GR class III (PTA $53.8 \mathrm{~dB}$, SDS $50 \%$ ). The patient did not report any subjective difference between her pre- and postoperative hearing.

\section{Discussion}

\section{Comparison Between the MCT and Other Monitoring Methods}

Intraoperative neurophysiological methods of moni- toring of the cochlear nerve are classified into two types: continuous monitoring to predict postoperative hearing function, and mapping to detect and trace the course of the nerve. Among the former types, ABR has most commonly been used. The problem with ABR, however, is that this method requires time for summation of acquisitions and is not able to trace the cochlear nerve anatomically and instantaneously. Instead of ABR, continuous monitoring of either the CNAP at the root entry zone or the

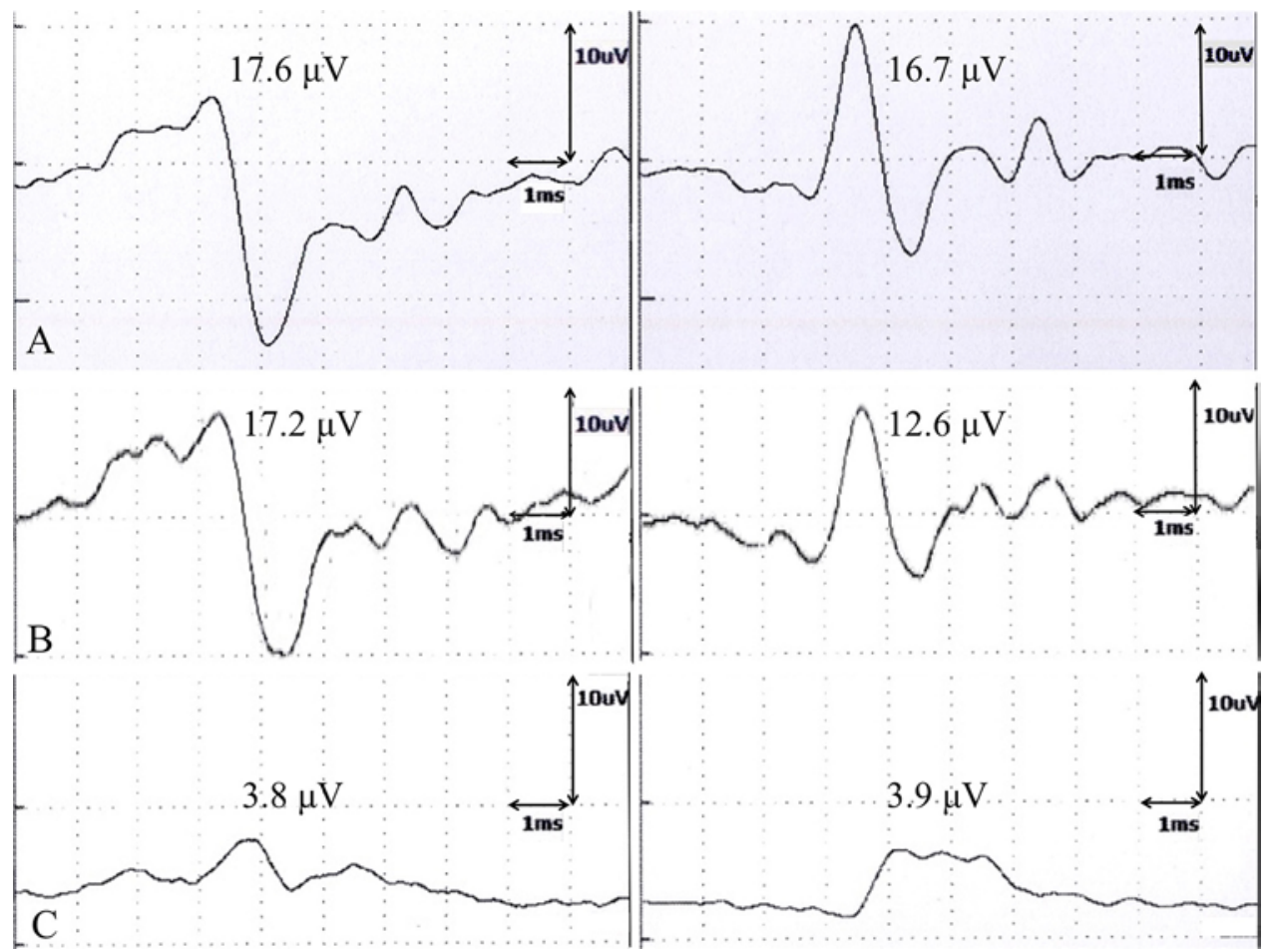

FIG. 4. Monopolar (left) and bipolar (right) recording with the MCT device in case 5. Recording locations included the root entry zone of the cochlear nerve $(\mathbf{A})$, the cisternal portion of the cochlear nerve $(B)$, and the cisternal portion of the inferior vestibular nerve $(\mathbf{C})$. Figure is available in color online only. 


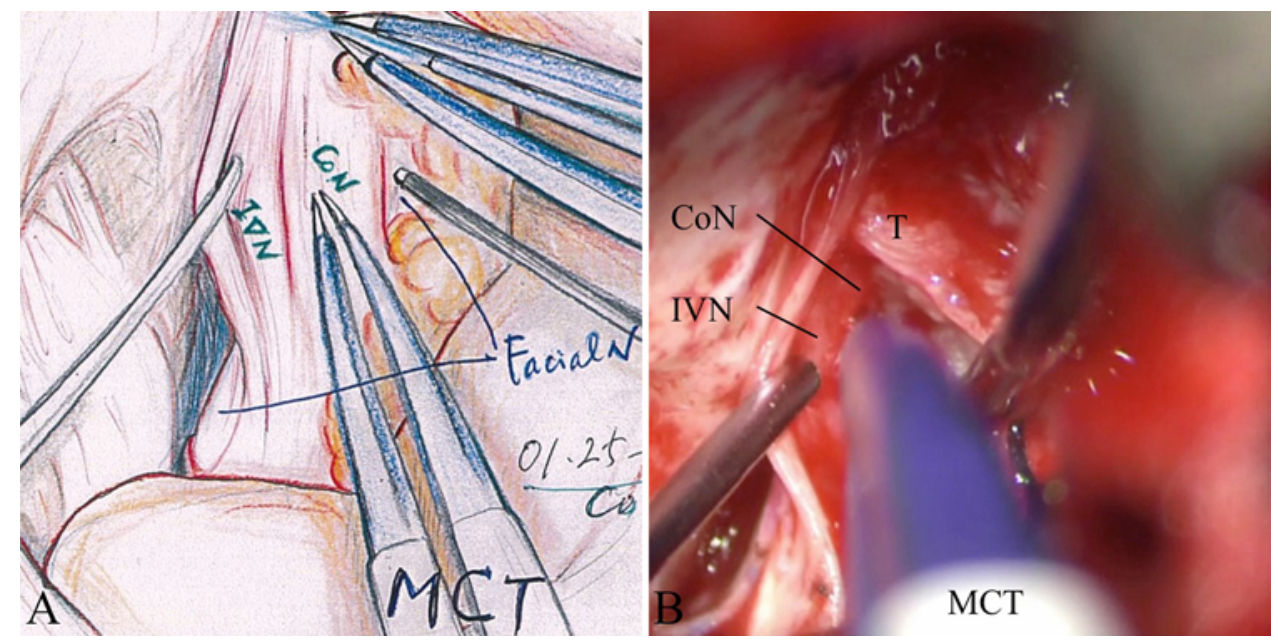

FIG. 5. Case 5. Operative note illustrated by the operator (A) and video capture (B). The MCT device was placed on the dorsal aspect of the cisternal portion of the cochlear nerve. The MCT recording is presented in Fig. 4B. Copyright Kazuhiko Fujitsu (panel A). Published with permission. Figure is available in color online only.

dorsal cochlear nucleus action potential has been reported to provide immediate feedback and successful prognostic assessment. ${ }^{10,12,17,18}$ However, in cases of large tumors it is very difficult at the early stage of operation to place an electrode in a deep location such as the root entry zone or the dorsal cochlear nucleus. Moreover, these methods use fixed, immobile electrodes. Dislocation of these electrodes during surgical manipulation may also cause a problem, because quantitative comparison is difficult between recordings in different locations. ${ }^{12}$ Some surgeons may be concerned that the placing of electrodes will interfere continuously with surgical manipulation.

Our newly designed MCT appears to solve these problems. It is easy to handle and can be used both on the sur- face and inside the tumor (Figs. 3 and 5) without interfering with surgical manipulation.

\section{Monopolar Versus Bipolar Recording}

Simultaneous monopolar and bipolar recording is possible with the use of the MCT. The amplitude of monopolar recording is usually larger than that of bipolar recording (Fig. 4A and B), indicating that the former has higher sensitivity than the latter. This is consistent with previous reports. ${ }^{3,11}$ In a report of direct cranial nerve VIII responses recorded in an animal experiment, monopolar responses were observable around $3 \mathrm{msec}$ on some structures other than the cochlear nerve: the vestibular nerve, the brainstem, and even the temporal bone. ${ }^{11}$ These re-

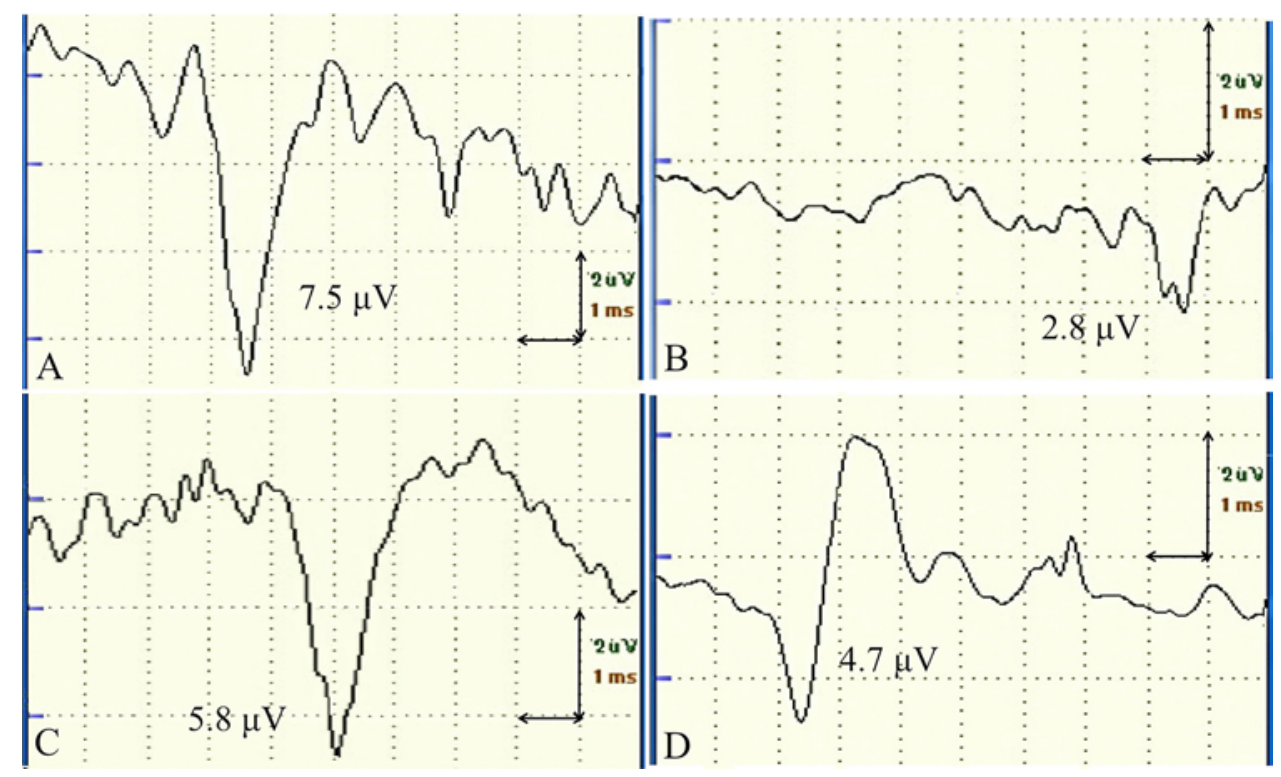

FIG. 6. Bipolar recording with the MCT device in case 18. Recording locations included the root entry zone of the presumed cochlear nerve $(A)$ and that of the presumed inferior vestibular nerve (B). Recordings on the tumor capsule very close to the cochlear nerve (C) and on the cochlear nerve after gross-total removal (D) are also shown. Figure is available in color online only. 
sponses were influenced by activity originating at remote sites, i.e., the cochlear nerve or the cochlear nucleus. This report suggested that the monopolar response is less location-specific than the bipolar response. As shown in Figs. $4 \mathrm{C}$ and $6 \mathrm{C}$, similar waveforms could also be seen in bipolar recording, but it is considered to be more location-specific than monopolar recording. The recordings in Figs. $4 \mathrm{C}$ and $6 \mathrm{C}$ show that responses from structures other than the cochlear nerve had lower amplitude and/or longer latency. Some investigators reported that bipolar recording exhibited sufficient specificity and selectivity for differentiating the cochlear nerve from other structures..$^{3,4,11,13}$ Moreover, considering that the operating room usually is an electrically noisy environment, bipolar recording is more desirable because a better signal-to-noise ratio can be obtained by noise subtraction. However, an important disadvantage of bipolar recording is that amplitude decrease can occur if the bipolar tips are placed obliquely onto the nerve axis. ${ }^{11}$ To avoid such an occurrence, the surgeon should place the bipolar tips parallel to the course of the nerve. When the nerve is fanned because of large tumor bulk and the bipolar tips are not placed parallel to the long axis of the nerve, the result can be influenced. Therefore, there is a possibility of false-positive and false-negative waveforms. A false-positive waveform can be caused by noise contamination, which may be suppressed by some ways to improve signal-to-noise ratio, including adjustment of filter range and summation times. On the other hand, a false-negative waveform can be caused by insufficient placement on the nerves, condition of the cochlear nerve, and sensitivity of the MCT. It is necessary to repeat probing while adjusting the delicate shifting on a fanning nerve and to develop a more sensitive electrode. Moreover, it is important to assess each waveform and to combine recording results with presumptive anatomical location of the nerves, to prevent false-positive and false-negative mapping results. We use the MCT mainly in the bipolar mode after a nerve or nervous structures have come into view under the surgical microscope. Until then, we sometimes use the monopolar mode to probe the cochlear nerve. The reason for this is, of course, its higher sensitivity.

\section{Actual Practice of MCT Mapping Combined With Microsurgical Presumption of the Nerve Location}

Before proceeding to MCT wave analysis, presumptive discrimination between the cochlear nerve and the vestibular nerves should be attempted under the surgical microscope. This presumption is made on the basis of the knowledge of normal anatomical arrangement of the nerves. A vestibular schwannoma always disrupts the arrangement and course of the nerves, but normal arrangement of each nerve still tends to remain near both the meatal and root entry or exit zone. After substantial debulking of tumor tissues, the remnants of the vestibular nerves were often able to be identified, except in cases 10,13 , and 15 in this series.

The most commonly observed arrangement of the nerves was the inferior vestibular nerve remnant presenting most caudally on the ventral surface of the tumor, and adjacent to and slightly ventrorostrally to this nerve was the cochlear nerve when the tumor probably originated mainly from the superior vestibular nerve (case 5, Figs. 3 and 5).

Keeping this anatomical arrangement in mind, when it is difficult for the surgeon to locate the cochlear nerve, frequent probing is advisable with the use of both more sensitive monopolar and more location-specific bipolar MCT. Whenever an unidentified nerve becomes visible under the microscope, repeated recording with the bipolar MCT should be performed on and around this nerve. The surgeon should keep repeating such recording on and on to the next or to the third unidentified nerve. By comparing these waveforms in terms of amplitude and latency, and by considering the anatomical arrangement of the nerves, the surgeon is eventually able to identify the cochlear nerve most of the time. It is known that the amplitude of the CNAP is variable, depending on where it is recorded in the course of the cochlear nerve. This implies that it is impossible to make a quantitative assessment, because the recording locations vary each time in intermittent recording with the MCT. An exact prediction of the hearing prognosis solely from changes in amplitude or waveform of the CNAP may be difficult. In case 5, bipolar recording from different portions of the cochlear nerve showed a slight difference in amplitude (Fig. 4A and B). Whether this difference is caused by the change of location, damage to the cochlear nerve, or oblique placement of the bipolar tips has not been determined in our studies so far. When the MCT is placed on a nerve structure and shows a waveform that has a lower amplitude or more prolonged latency compared with the typical CNAP (Figs. 4C and 6B), the possibility of a vestibular nerve remnant should always be considered. In such a situation, knowledge of the normal arrangement of the nerves may help discriminate between the nerves. Recording on the tumor capsule very close to the cochlear nerve resulted in only a slight prolongation of latency (Fig. 6C). These findings pose difficult issues to analyze comprehensively, but we think that definite differences in amplitude or in latency have differential diagnostic significance when compared with the cochlear nerve response.

\section{Cases of Complete Mapping Failure or Discrepancies Between Anatomical Preservation and Hearing Function}

As for the positive predictive value of MCT, 10 (77\%) of 13 cases in which mapping was successful throughout the operation resulted in useful hearing. In 3 exceptional cases (cases 7, 16, and 18), cochlear nerve mapping was successful throughout the operation but their GR classes were downgraded postoperatively. In cases 16 and 18 (from GR class II to III), although their GR classes themselves were downgraded, substantial hearing function was preserved. In case 7 (from GR class II to IV), almost the entire course of the cochlear nerve was able to be traced; however, the nerve was technically damaged during tumor removal. These cases were not considered to be errors in identifying the cochlear nerve. In brief, we think that these did not correspond to false-positive mapping and did not reduce the positive predictive value of MCT.

As for the negative predictive value, $4(80 \%)$ of 5 cases in which MCT failed to detect and trace the cochlear nerve resulted in hearing loss. The only exceptional case (case 
13), in which MCT failed to detect CNAP but hearing function was preserved, was likely to be a false-negative identification. We speculate in this case that the cochlear nerve was not able to be detected, but was unexpectedly preserved ventral to the vestibular nerve remnant tissues.

After closely reviewing the microsurgical findings in these cases, we assume that whether the tumor originated from the superior or inferior vestibular nerve influences both successful mapping and hearing preservation. We speculate that tumors originating from the inferior vestibular nerve may have an increased risk of hearing loss compared with those originating from the superior vestibular nerve. When the tumor appeared to originate from the superior vestibular nerve, cochlear nerve mapping with MCT was almost always successful and hearing function was preserved (cases 1, 2, 3, 5, 8, 9, 11, 12, 14, and 17). Even when the tumor origin was the superior vestibular nerve, preservation of the cochlear nerve was not always successfully affected by surgical mishandling (cases 4 and $6)$. When the tumor seemed to originate from the inferior vestibular nerve, the cochlear nerve was easily injured and hearing acuity tended to deteriorate to a certain degree (cases 7, 16, and18). Certainly, a refined microsurgical dissection technique is of primary importance to preserve the cochlear nerve in either case.

When both vestibular nerve remnants are spread over the dorsal surface of the tumor, the cochlear nerve is difficult to identify and may be sandwiched between the (inferior) vestibular nerve remnant and the tumor tissues or may be isolated far ventrally. MCT easily failed to detect CNAP even in small tumors (cases 10 and 13).

When the tumor was deeply embedded in the brainstem involving the exit zone of the facial nerve and the entry zone of the acoustic nerve complex, the cochlear nerve was extremely difficult to detect and MCT often failed to give any reliable response. Facial nerve damage also occurred easily in this situation (case 15).

The rate of hearing preservation after surgery for large tumors $(>20 \mathrm{~mm})$ in the literature is $9.1 \%-27.5 \%{ }^{16}$ Generally speaking, it is thought to be more difficult to preserve hearing function in cases of larger tumors. ${ }^{14,15,19,20}$ Some researchers argue that the extracanalicular length of tumor-cochlear nerve contact increases the risk of postoperative hearing loss. ${ }^{20}$

Regarding facial nerve preservation, some authors reported that the method of mapping combined with intermittent stimulation and continuous monitoring in the root exit zone of the facial nerve was most helpful to preserve good function of the facial nerve. ${ }^{1}$ Taking this report into consideration, our MCT, if combined with continuous CNAP monitoring in the root entry zone of the cochlear nerve (which becomes possible only after substantial debulking of the tumor), may increase the chances of hearing preservation.

\section{Conclusions}

Our newly developed MCT device proved to be useful in cochlear nerve identification and mapping in hearingpreservation VSS. In addition to the precise analysis of the anatomical arrangement of the nerves, including the ves- tibular nerve remnants, frequent probing on and around an unidentified nerve and comparison of each waveform are advisable with the use of both more sensitive monopolar and more location-specific bipolar MCT. This device may consequently be helpful in hearing preservation but at the same time has some limitations.

\section{References}

1. Amano M, Kohno M, Nagata O, Taniguchi M, Sora S, Sato $\mathrm{H}$ : Intraoperative continuous monitoring of evoked facial nerve electromyograms in acoustic neuroma surgery. Acta Neurochir (Wien) 153:1059-1067, 2011

2. Butler S, Coakham H, Maw R, Morgan H: Physiological identification of the auditory nerve during surgery for acoustic neuroma. Clin Otolaryngol Allied Sci 20:312-317, 1995

3. Colletti V, Fiorino FG: Bipolar recording of the cochlear nerve action potentials during cerebellopontine angle surgery. Am J Otol 15:798-803, 1994

4. Colletti V, Fiorino FG: Electrophysiologic identification of the cochlear nerve fibers during cerebello-pontine angle surgery. Acta Otolaryngol 113:746-754, 1993

5. Danner C, Mastrodimos B, Cueva RA: A comparison of direct eighth nerve monitoring and auditory brainstem response in hearing preservation surgery for vestibular schwannoma. Otol Neurotol 25:826-832, 2004

6. Gardner G, Robertson JH: Hearing preservation in unilateral acoustic neuroma surgery. Ann Otol Rhinol Laryngol 97:55-66, 1988

7. House JW, Brackmann DE: Facial nerve grading system. Otolaryngol Head Neck Surg 93:146-147, 1985

8. Koos WT, Day JD, Matula C, Levy DI: Neurotopographic considerations in the microsurgical treatment of small acoustic neurinomas. J Neurosurg 88:506-512, 1998

9. Møller AR, Jannetta PJ: Compound action potentials recorded intracranially from the auditory nerve in man. Exp Neurol 74:862-874, 1981

10. Nakatomi H, Miyazaki H, Tanaka M, Kin T, Yoshino M, Oyama H, et al: Improved preservation of function during acoustic neuroma surgery. J Neurosurg 122:24-33, 2015

11. Nguyen BH, Javel E, Levine SC: Physiologic identification of eighth nerve subdivisions: direct recordings with bipolar and monopolar electrodes. Am J Otol 20:522-534, 1999

12. Piccirillo E, Hiraumi H, Hamada M, Russo A, De Stefano A, Sanna M: Intraoperative cochlear nerve monitoring in vestibular schwannoma surgery-does it really affect hearing outcome? Audiol Neurotol 13:58-64, 2008

13. Rosenberg SI, Martin WH, Pratt H, Schwegler JW, Silverstein H: Bipolar cochlear nerve recording technique: a preliminary report. Am J Otol 14:362-368, 1993

14. Samii M, Gerganov V, Samii A: Improved preservation of hearing and facial nerve function in vestibular schwannoma surgery via the retrosigmoid approach in a series of $200 \mathrm{pa}-$ tients. J Neurosurg 105:527-535, 2006

15. Samii M, Matthies C: Management of 1000 vestibular schwannomas (acoustic neuromas): hearing function in 1000 tumor resections. Neurosurgery 40:248-262, 1997

16. Wanibuchi M, Fukushima T, McElveen JT Jr, Friedman AH: Hearing preservation in surgery for large vestibular schwannomas. J Neurosurg 111:845-854, 2009

17. Yamakami I, Oka N, Yamaura A: Intraoperative monitoring of cochlear nerve compound action potential in cerebellopontine angle tumour removal. J Clin Neurosci 10:567-570, 2003

18. Yamakami I, Yoshinori H, Saeki N, Wada M, Oka N: Hearing preservation and intraoperative auditory brainstem response and cochlear nerve compound action potential monitoring in the removal of small acoustic neurinoma via 
the retrosigmoid approach. J Neurol Neurosurg Psychiatry 80:218-227, 2009

19. Yates PD, Jackler RK, Satar B, Pitts LH, Oghalai JS: Is it worthwhile to attempt hearing preservation in larger acoustic neuromas? Otol Neurotol 24:460-464, 2003

20. Yong RL, Westerberg BD, Dong C, Akagami R: Length of tumor-cochlear nerve contact and hearing outcome after surgery for vestibular schwannoma. J Neurosurg 108:105-110, 2008

\section{Disclosures}

The authors report no conflict of interest concerning the materials or methods used in this study or the findings specified in this paper.

\section{Author Contributions}

Conception and design: Watanabe, Ishii, Fujitsu, Kaku. Acqui- sition of data: Watanabe. Analysis and interpretation of data: Watanabe, Ishii, Fujitsu. Drafting the article: Watanabe. Critically revising the article: Ishii, Fujitsu. Reviewed submitted version of manuscript: Murayama. Study supervision: Ichikawa, Miyahara, Okada, Tanino, Uriu.

\section{Supplemental Information}

\section{Previous Presentations}

This paper was presented at the 21st Annual Meeting of the Japanese Congress for Brain Tumor Surgery, Toranomon, Tokyo, Japan, September 9, 2016, and at the 29th Annual Meeting of the Japanese Society for Skull Base Surgery, Matsumoto, Nagano, Japan, June 16, 2017.

\section{Correspondence}

Nobuyuki Watanabe: National Hospital Organization, Yokohama Medical Center, Kanagawa, Japan.ms05-watanabeno@hotmail. co.jp. 